\title{
Diffuse Pulmonary Lymphangiomatosis
}

National Cancer Institute

\section{Source}

National Cancer Institute. Diffuse Pulmonary Lymphangiomatosis. NCI Thesaurus. Code C45630.

Lymphangiomatosis diffusely involving the lung parenchyma. 